\title{
PRODUCCIÓN CIENTÍFICA DE ANDALUCÍA EN LAS BASES DE DATOS SCIENCE CITATION INDEX E ÍNDICE ESPAÑOL DE CIENCIA Y TECNOLOGÍA
}

\author{
Carmen Urdín y Fernanda Morillo*
}

Resumen: Se analiza la producción científica de Andalucía recogida en las bases de datos Science Citation Index (SCI) e Indice Español de Ciencia y Tecnología (ICYT). Se muestra el número total de documentos de Andalucía en ambas bases de datos, el porcentaje que dichos documentos representan respecto a España, la distribución de los documentos por provincias, áreas temáticas abordadas y sectores institucionales, el análisis pormenorizado del sector universitario y las revistas más productivas.

Palabras clave: producción científica, Andalucía, bases de datos, SCI, ICYT, estudio bibliométrico.

Abstracts: This study analyses the scientific production of Andalucía as seen through Science Citation Index (SCI) and Science and Technology Spanish Index (ICYT) databases. Total number of documents of Andalucía, percentage of total Spanish production, geographic distribution, subject categories, institutional sectors (University detailed) and most productive journals are examined through both databases.

Keywords: scientific production, Andalucía, databases, SCI, ICYT, bibliometric study.

\section{Introducción}

Un amplio estudio que analizaba la producción científica en Andalucía (1) publicado en el año 1995, daba a conocer dicha producción en las bases de datos del Institute for Scientific Information de Filadelfia (ISI). Es sabido que las bases de datos bibliográficas desarrolladas por el ISI recogen preferentemente revistas de habla inglesa y sobre todo aquéllas que difunden la ciencia básica frente a la aplicada y el área biomédica frente a la tecnológica. Para contemplar un estudio de producción científica completo habría que cuantificar también la literatura científica publicada en aquellas revistas que no están recogidas en las bases de datos del ISI pero sí lo están en las bases de datos propias de cada país.

El presente trabajo tiene como finalidad dar a conocer la producción científica en Ciencia y Tecnología de la Comunidad Andaluza, publicada tanto en revistas españolas como extranjeras a través de dos bases de datos. Las bases de datos utilizadas son el Índice Español de Ciencia y Tecnología (ICYT (2), de cobertura nacional, período 1979-junio 1997, y Science Citation Index (SCI (3), de cobertura internacional, perfodo 1990-1997). La complementariedad que presentan estas bases de datos nos pue-

\footnotetext{
* Centro de Información y Documentación Cientifica (CINDOC), CSIC.

Correo-e: curdin@cindoc.csic.es

Recibido: Primera versión: 23-6-99. Segunda versión: 14-8-2000.
} 
de acercar a conocer la producción total sobre los resultados de la investigación que se lleva a cabo en dicha Comunidad $(4,5)$. Con ello se pretende analizar cuantitativamente la visibilidad internacional y nacional en las diversas áreas temáticas. Tratándose de períodos distintos, el tipo de comparación que se establece es porcentual y lo que se pretende fundamentalmente es tener una visión global de la producción científica andaluza.

\section{Metodología}

El Science Citation Index (SCI) es una base de datos bibliografica producida por el Institute for Scientific Information (ISI) de Filadelfia, EE.UU. El SCI emplea las referencias citadas en los documentos publicados como términos temáticos, relacionándolos entre sí y facilitando la búsqueda multidisciplinar. Esta base de datos presenta la ventaja principal de tener un carácter multidisciplinar e internacional, ya que realiza un vaciado total de unas 3.200 revistas de gran difusión internacional («main stream science») en ciencias experimentales y medicina en su versión en CD-ROM. Dicho vaciado permite el estudio pormenorizado de instituciones, autores y colaboraciones entre ellos, ya que el SCI recoge la información correspondiente a todos los autores e instituciones firmantes de los documentos. En este estudio, para facilitar la comparación con la base de datos ICYT que no recoge artículos sobre Medicina, se ha prescindido de la parte de Medicina clínica recogida en el SCI.

El Índice Español de Ciencia y Tecnología (ICYT), base de datos desarrollada por el Consejo Superior de Investigaciones Científicas (CSIC), tiene como finalidad principal recopilar y difundir la producción científica española publicada en revistas de nuestro país, la cual está recogida, en general, de forma incompleta por las bases de datos internacionales. Por el contenido de sus registros y las posibilidades de recuperación por todos sus campos, constituye también una herramienta excelente para estudiar la aportación a la literatura científica española de una institución, de un centro específico, o de una zona geográfica determinada $(6,7)$. Esta base de datos cubre las áreas de ciencia y tecnología y analiza unas 500 publicaciones periódicas y no periódicas españolas. No hay solapamiento entre ambas bases de datos, exceptuando la revista Anales de Química que está recogida en las 2 bases de datos y en el presente estudio se ha contabilizado sólo en ICYT.

Los datos que se extrajeron del SCI en formato CD-ROM, período 1990-97, se obtuvieron buscando «Spain» en el campo «address». Dicha selección se descargó en una base de datos de ficheros relacionales en $\mathrm{dBaseV}$, diseñada a tal efecto y posteriormente tratada en Access97. Consta de tres ficheros de datos: uno con información general de los documentos, otro de autores y otro de instituciones, relacionados entre sí a través del número de identificación. Las instituciones hubieron de homologarse y codificarse para poder realizar el estudio pormenorizado de las mismas. La homologación se hizo de forma semiautomática asignando el código de provincia y otro código identificador de los sectores institucionales. Del conjunto de documentos así tratados, se seleccionaron aquéllos en los que figuraban las provincias andaluzas como ubicación del centro de trabajo.

Para la selección de los artículos publicados por la Comunidad Autónoma Andaluza en la base de datos ICYT (período 1979-Jun.1997) se elaboró una cadena o per- 
fil de búsqueda que contiene los términos Andalucía o los nombres de las ocho provincias andaluzas, realizando la búsqueda en el campo «organismo».

Para poder abordar el estudio de la temática tratada en los documentos obtenidos y dado que las dos bases de datos presentan diferente clasificación temática, se han tenido en cuenta las características de cada una, para intentar unificar los resultados. Así, la base de datos SCI clasifica cada revista hasta en 5 disciplinas diferentes, según una clasificación elaborada por dicha base de datos y la base de datos ICYT clasifica cada documento al nivel de subdisciplina, siguiendo la Nomenclatura Internacional de la UNESCO para la Ciencia y Tecnología. Ante esta diferencia, la tabla de las clasificaciones que se ha elaborado se ha ajustado a los campos científicos UNESCO.

Los sectores institucionales considerados son los siguientes: Administración Central, asociaciones y colegios profesionales, fundaciones sin ánimo de lucro, CSIC, empresas, Junta de Andalucía y Universidad. Las universidades recogen su producción y, además, la propia de los centros mixtos CSIC-Universidad. CSIC también incluye esta producción conjunta.

\section{Resultados}

Las cifras de producción científica de la Comunidad Autónoma de Andalucía en la base de datos SCI es de 12.530 documentos y en la base de datos ICYT es de 11.070 documentos. Estos valores vienen a representar entre un $13-14 \%$ del total de la producción española. Considerando que los valores obtenidos de ICYT representan la producción científica publicada en revistas españolas y los datos del SCI la publicada en revistas internacionales, se puede apreciar que la comunidad científica y tecnológica andaluza, en conjunto, publica sus trabajos en ambos medios.

\section{Provincias}

Dentro de Andalucía, la producción científica de documentos se reparte del siguiente modo entre sus provincias: Granada y Sevilla son las más productivas, con más del $30 \%$ del total, seguidas por Córdoba y Málaga (tabla I). Hay que tener en cuenta que la producción de las diferentes provincias e instituciones se solapa debido a las colaboraciones y, por tanto, en las tablas siguientes, la suma total de documentos es mayor que el número de documentos reales obtenidos.

\section{Temática}

Se ha analizado la producción de Andalucía en las grandes áreas de Ciencia y Tecnología. Los resultados obtenidos de ambas bases de datos se han adaptado al mismo sistema de clasificación que se corresponde con los códigos UNESCO, al nivel de campo científico (tabla II).

Siguiendo la distribución de esta tabla, en la figura 1 se muestra la producción de documentos de Andalucía en la base de datos SCI y la producción en la base de da- 
Tabla I

Producción científica de Andalucía, desglosada por provincias

\begin{tabular}{|l|rr|rr|rr|}
\hline & \multicolumn{2}{|c|}{ SCI } & \multicolumn{2}{c|}{ ICYT } & \multicolumn{2}{c|}{ Total } \\
\hline Provincias & $N^{\circ}$ doc. & \% & N. ${ }^{\circ}$ doc. & \% & N. ${ }^{\circ}$ doc. & \% \\
\hline Granada & 4.126 & 32,9 & 3.772 & 34,1 & 7.898 & 33,5 \\
Sevilla & 4.202 & 33,5 & 3.215 & 29,0 & 7.417 & 31,4 \\
Cóndoba & 2.036 & 16,2 & 2.120 & 19,2 & 4.156 & 17,6 \\
Málaga & 1.379 & 11,0 & 1.182 & 10,7 & 2.561 & 10,9 \\
Cádiz & 712 & 5,7 & 684 & 6,2 & 1.396 & 5,9 \\
Almería & 385 & 3,1 & 356 & 3,2 & 741 & 3,1 \\
Jaén & 291 & 2,3 & 406 & 3,7 & 697 & 3,0 \\
Huelva & 118 & 0,9 & 232 & 2,1 & 350 & 1,5 \\
\hline
\end{tabular}

Tabla II

Clasificación utilizada

\begin{tabular}{|c|c|c|}
\hline Clasificación UNESCO & Area temática SCI & Area temática ICYT \\
\hline Ciencias Agrarias & Agricultura & Ciencias Agrarias \\
\hline Ciencias Tecnológicas & $\begin{array}{l}\text { Ingeniería y Tecnología + } \\
\text { Ciencia de Materiales }\end{array}$ & Ciencias Tecnologicas \\
\hline Ciencias de la Tierra y del Espacio & Ciencias de la Tierra & Ciencias de la Tierra y del Espacio \\
\hline Ciencias de la Vida & $\begin{array}{l}\text { Biologfa descriptiva + } \\
\text { Investigación biomédica }\end{array}$ & $\begin{array}{l}\text { Ciencias de la Vida + } \\
\text { Farmacología + Toxicologia }\end{array}$ \\
\hline Física + Astronomía y Astrofísica & $\begin{array}{l}\text { Física (incluye } \\
\text { Astronomía y Astrofísica) }\end{array}$ & Física + Astronomía y Astrofísica \\
\hline Matemáticas & Matemáticas & Matemáticas \\
\hline Multidisciplinar & Multidisciplinar & - \\
\hline Química & Química & Química \\
\hline
\end{tabular}

tos ICYT según su temática. En ella se observa que en el SCI destacan las áreas de Ciencias de la Vida, que es el área más productiva (con un $46 \%$ del total), seguida por Química (24\%) y Física (14\%). En la base de datos española ICYT, aunque destaca también el área de Ciencias de la Vida (36\% del total de documentos), las siguientes áreas no se corresponden con las anteriores y son las Ciencias Tecnológicas (22\%), Ciencias Agrarias (18\%) y Ciencias de la Tierra (15\%) las áreas que publican preferentemente en revistas españolas. Esta distribución es lógica si se piensa que la ciencia básica (Física, Química, Ciencias de la Vida) tiene un carácter más internacional que la aplicada (Ciencias Tecnológicas, Ciencias Agrarias, Ciencias de la Tierra). 
Figura 1

Producción científica de artículos de Andalucía, desglosada por áreas temáticas

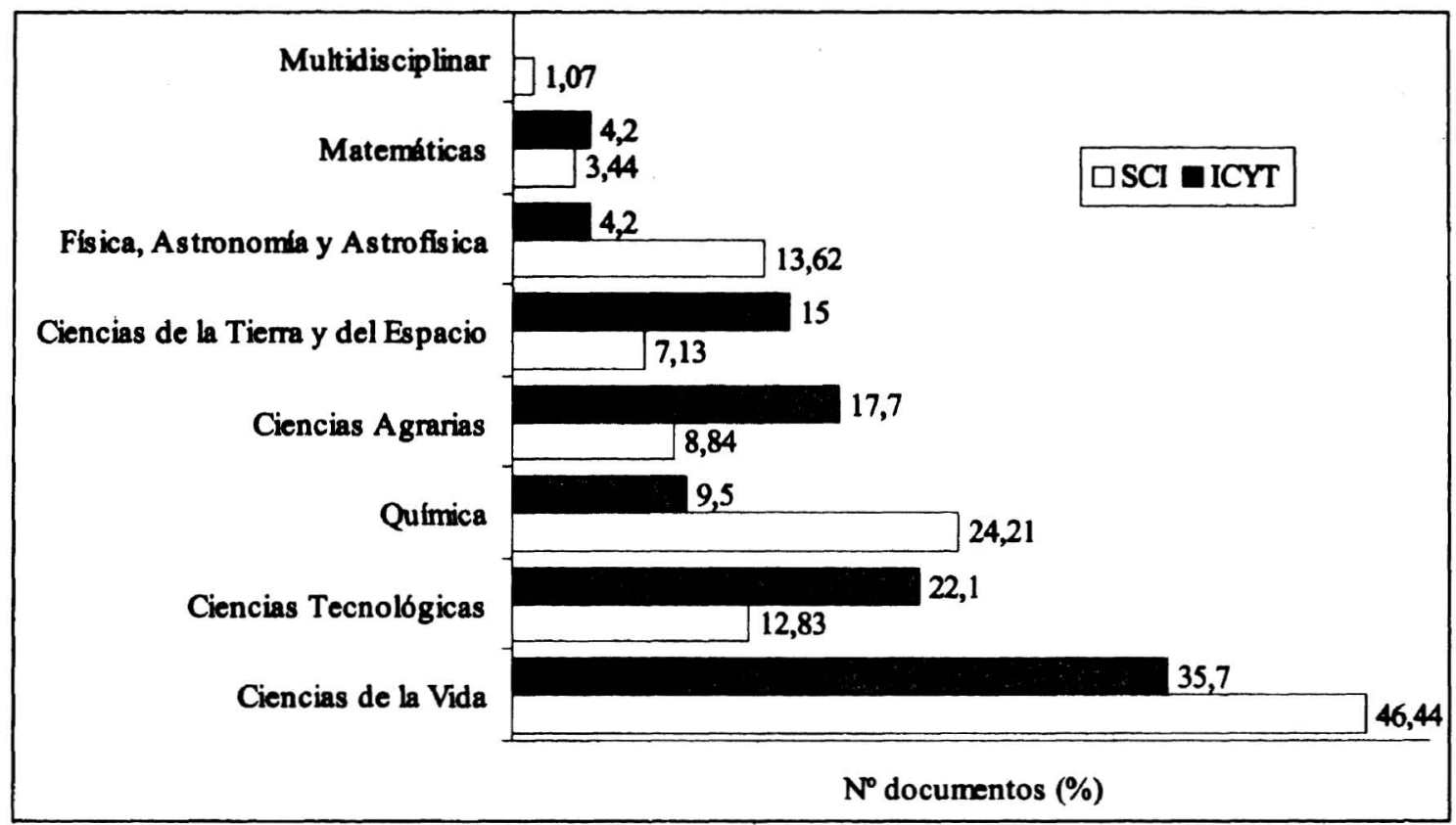

\section{Sectores institucionales}

La producción científica correspondiente a los sectores institucionales muestra, en parte, considerables parecidos entre las bases datos SCI e ICYT (tabla III). La Universidad aporta en ambos casos más del $75 \%$ de la producción científica ( $80 \%$ en SCI y $77 \%$ en ICYT). El CSIC representa un $22 \%$ de la producción andaluza en las dos bases de datos. Estos valores nos indican que estas dos instituciones utilizan tanto revistas españolas como internacionales para la publicación de sus trabajos. El valor tan elevado correspondiente a la Universidad es un claro signo de que en Andalucía la investigación que se realiza procede mayoritariamente del sector universitario.

Si comparamos estos valores de producción científica de la Universidad y del CSIC en Andalucía, obtenidos de la base de datos SCI, con los datos de producción de estas instituciones a escala nacional, se observa que la Universidad en Andalucía supera el porcentaje de la producción en España que es de $64 \%$, y lo publicado por el CSIC en Andalucía está dentro de los mismos valores que para el total de España $(21 \%)$.

La tercera posición la ocupan los organismos que pertenecen administrativamente a la Junta de Andalucía con un porcentaje similar en las dos bases de datos analizadas $(8 \%)$.

Las siguientes posiciones en cuanto a producción científica las ocupan los organismos que dependen de la Administración Central, siendo el valor obtenido del SCI bastante inferior al de ICYT, lo que nos indica que los científicos y tecnólogos de estas instituciones publican preferentemente en revistas españolas. A continuación, con un bajo porcentaje de participación en ambas bases de datos, se encuentran las Em- 
presas (pertenecientes en su mayoría al sector agroalimentario). La producción correspondiente al sector empresarial obtenida en el SCI concuerda con los valores relativos a España y a la Comunidad de Madrid, publicados en otros informes (8). Sin embargo, en ICYT este valor es bastante inferior a lo producido por las empresas de la Comunidad de Madrid, que es del orden del $26 \%$, dato que se explica porque la ubicación de empresas se encuentra en núcleos de poder económico como Madrid. En último lugar se encuentran los Colegios y Asociaciones profesionales cuya aportación en ambas bases de datos es puramente testimonial.

Tabla III

Producción científica de Andalucía desglosada por sectores institucionales

\begin{tabular}{|l|rc|rc|rc|}
\hline & \multicolumn{2}{|c|}{ SCI } & \multicolumn{2}{c|}{ ICYT } & \multicolumn{2}{c|}{ Total } \\
\hline Sector institucional & $N^{\circ}$ doc. & $\%$ & $N^{\circ}$ doc. & $\%$ & $N^{\circ}$ doc. & $\%$ \\
\hline Universidad & 10.081 & 80,5 & 8.545 & 77,2 & 18.626 & 78,9 \\
CSIC & 3.086 & 24,6 & 2.188 & 19,8 & 5.274 & 22,3 \\
Junta de Andalucía & 1.077 & 8,6 & 822 & 7,4 & 1.899 & 8 \\
Administración Central & 317 & 2,5 & 803 & 7,3 & 1.120 & 4,7 \\
Empresas & 152 & 1,2 & 389 & 3,5 & 541 & 2,3 \\
Colegios y Asociaciones prof. & 20 & 0,2 & 21 & 0,2 & 41 & 0,2 \\
No consta & 103 & 0,8 & - & - & - & - \\
\hline
\end{tabular}

Si se observa la distribución temática de los 16 centros de ciencia y tecnología del CSIC en Andalucía (tabla IV), se comprende la importancia de la producción en las áreas biomédicas pero, sobre todo, en las áreas tecnológicas, agrarias y de la tierra.

Para las 8 universidades andaluzas también es interesante hacer un estudio pormenorizado, sobre todo debido a su mayor producción científica.

La aportación tan destacada de la Universidad hace oportuno un análisis especifico de la misma. Al analizar estas aportaciones hay que tener en cuenta la desigual antiguiedad de las universidades andaluzas, pues aunque el período estudiado es bastante actual, dicha antigüedad se manifiesta en el número de facultades que tienen, y en consecuencia en el de sus profesores, que son los principales productores de publicaciones.

En la tabla V se presentan los datos correspondientes al año de creación de las distintas universidades, así como el número de profesores y de alumnos que las constituyen.

La universidad más antigua es la de Sevilla, seguida de la de Granada, si bien ambas universidades tenían centros dependientes en otras provincias; así la Universidad de Sevilla tenía centros en Huelva, y la de Granada en Almería y Jaén, hasta la creación de éstas en 1993.

En la tabla VI se observa la producción de las universidades andaluzas destacando la de Granada (con un $27 \%$ ), Sevilla (21\%), Córdoba (16\%) y Málaga (9\%). Si 
analizamos por separado en cada base de datos la producción de las universidades que más han contribuido a la producción científica de Andalucía, hay que destacar que las universidades de Granada y de Córdoba han publicado más en revistas españolas; en cambio la Universidad de Sevilla lo ha hecho mayoritariamente en revistas extranjeras.

\section{Tabla IV}

\section{Centros del CSIC en Andalucía, por áreas de pertenencia}

Biología y Blomedicina

- Instituto de Bioquímica Vegetal y Fotosíntesis (IBVF)

- Instituto de Parasitología y Biomedicina 'López Neyra' (IPLN)

Ciencia y Tecnología de Alimentos

- Instituto de la Grasa (IG)

Ciencia y Tecnologías Físicas

- Centro Nacional de Aceleradores (CNA)

- Instituto de Astrofísica de Andalucía (IAA)

- Instituto de Microelectrónica de Sevilla (IMS-CNM)

Ciencia y Tecnología de Materiales

- Instituto de Ciencia de Materiales de Sevilla (ICMS)

Ciencia y Tecnologías Químicas

- Instituto de Investigaciones Químicas (IIQ)

Ciencias Agrarias

- Estación Experimental del Zaidín (EEZ)

- Estación Experimental «La Mayora» (EELM)

- Instituto de Agricultura Sostenible (IAS)

- Instituto de Recursos Naturales y Agrobiología (IRNAS)

Recursos Naturales

- Estación Biológica de Doñana (EBD)

- Estación Experimental de Zonas Aridas (EEZA)

- Instituto Andaluz de Ciencias de la Tierra (IACT)

- Instituto de Ciencias Marinas de Andalucía (ICMAN)

Tabla V

Datos relativos a las universidades andaluzas

\begin{tabular}{|l|c|c|c|}
\hline \multicolumn{1}{|c|}{ Universidad } & Año & $N^{\circ}$ profesores & $N .^{\circ}$ alumnos \\
\hline Sevilla & 1505 & 3.045 & 68.448 \\
Granada & 1531 & 2.932 & 61.448 \\
Córdoba & 1972 & 1.131 & 19.088 \\
Málaga & 1979 & 1.438 & 32.995 \\
Cádiz & 1993 & 1.211 & 19.296 \\
Jaén & 1993 & Sin datos & 12.050 \\
Almería & 1993 & Sin datos & 9.420 \\
Huelva & 1993 & 489 & 10.940 \\
\hline
\end{tabular}

Fuente: Gura de la Universidad 1994/1995 (9). 
Tabla VI

Producción cientínca de la Universidad.

\begin{tabular}{|l|rr|rr|rr|}
\hline & \multicolumn{2}{|c|}{$S C I$} & \multicolumn{2}{c|}{ ICYT } & \multicolumn{2}{c|}{ Total } \\
\hline Universidad & $N^{\circ} .^{\circ}$ doc. & $\%$ & $N^{\circ}$ doc. & $\%$ & $N^{\circ}$ doc. & $\%$ \\
\hline Granada & 3.058 & 24,4 & 3.312 & 29,9 & 6.370 & 27,0 \\
Sevilla & 2.977 & 23,8 & 2.030 & 18,3 & 5.007 & 21,2 \\
Córdoba & 1.851 & 14,8 & 1.887 & 17 & 3.738 & 15,8 \\
Málaga & 1.242 & 9,9 & 914 & 8,3 & 2.156 & 9,1 \\
Cádiz & 580 & 4,6 & 386 & 3,5 & 966 & 4,1 \\
Jaén & 284 & 2,3 & 345 & 3,1 & 629 & 2,7 \\
Almería & 283 & 2,3 & 145 & 1,3 & 428 & 1,8 \\
Huelva & 79 & 0,6 & 156 & 1,4 & 235 & 1,0 \\
\hline
\end{tabular}

En las figuras 2-9 se presenta la distribución temática de la producción de las ocho universidades destacando: Ciencias de la Vida (para Cádiz, Córdoba y Sevilla en la base de datos SCI, para Jaén en la base de datos ICYT y para Granada y Málaga en las dos bases de datos), Química (para Almería y Jaén en la base de datos SCI), Ciencias Agrarias (para Almería y Córdoba en la base de datos ICYT), Ciencias Tecnológicas (para Cádiz y Sevilla en la base de datos ICYT), Ciencias de la Tierra y del Espacio (para Huelva en las dos bases de datos). Obsérvese que, al igual que en la figura 1, las ciencias básicas se encuentran más representadas en la base de datos SCI y las aplicadas en la base de datos ICYT.

Figura 2.

Distribución temática de documentos de la Universidad de Almería (en porcentaje)

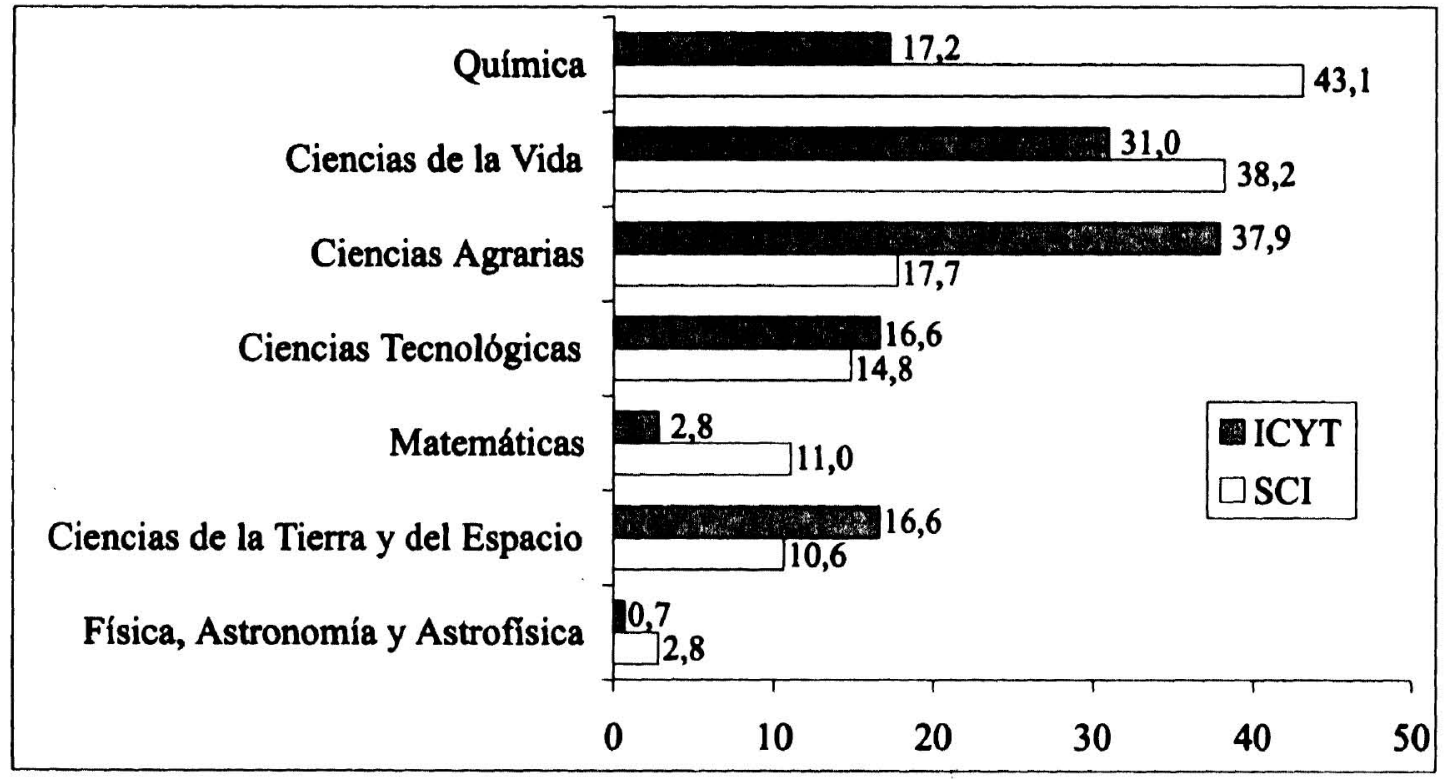




\section{Figura 3}

Distribución temática de documentos de la Universidad de Cádiz (en porcentaje)

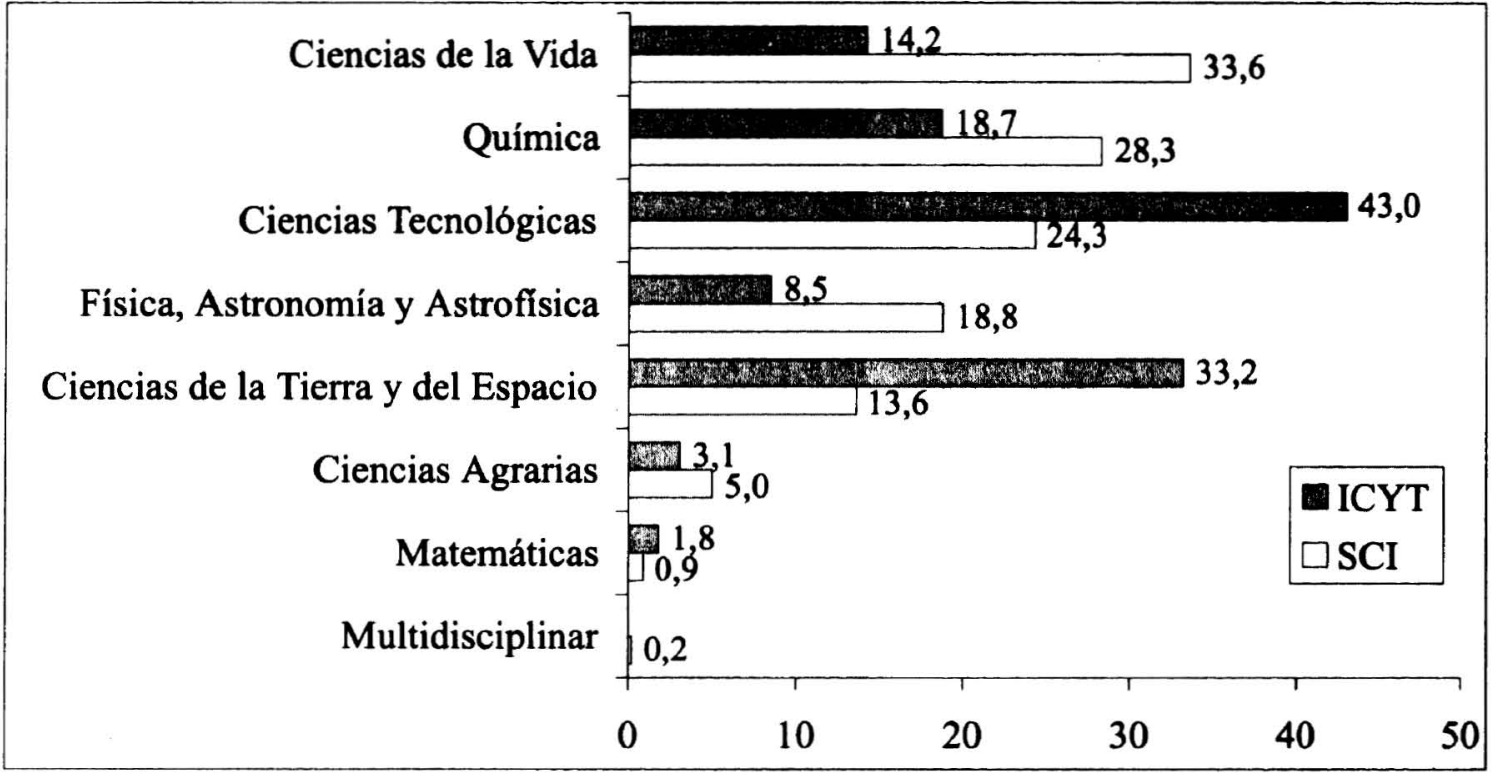

Figura 4

Distribución temática de documentos de la Universidad de Córdoba (en porcentaje)

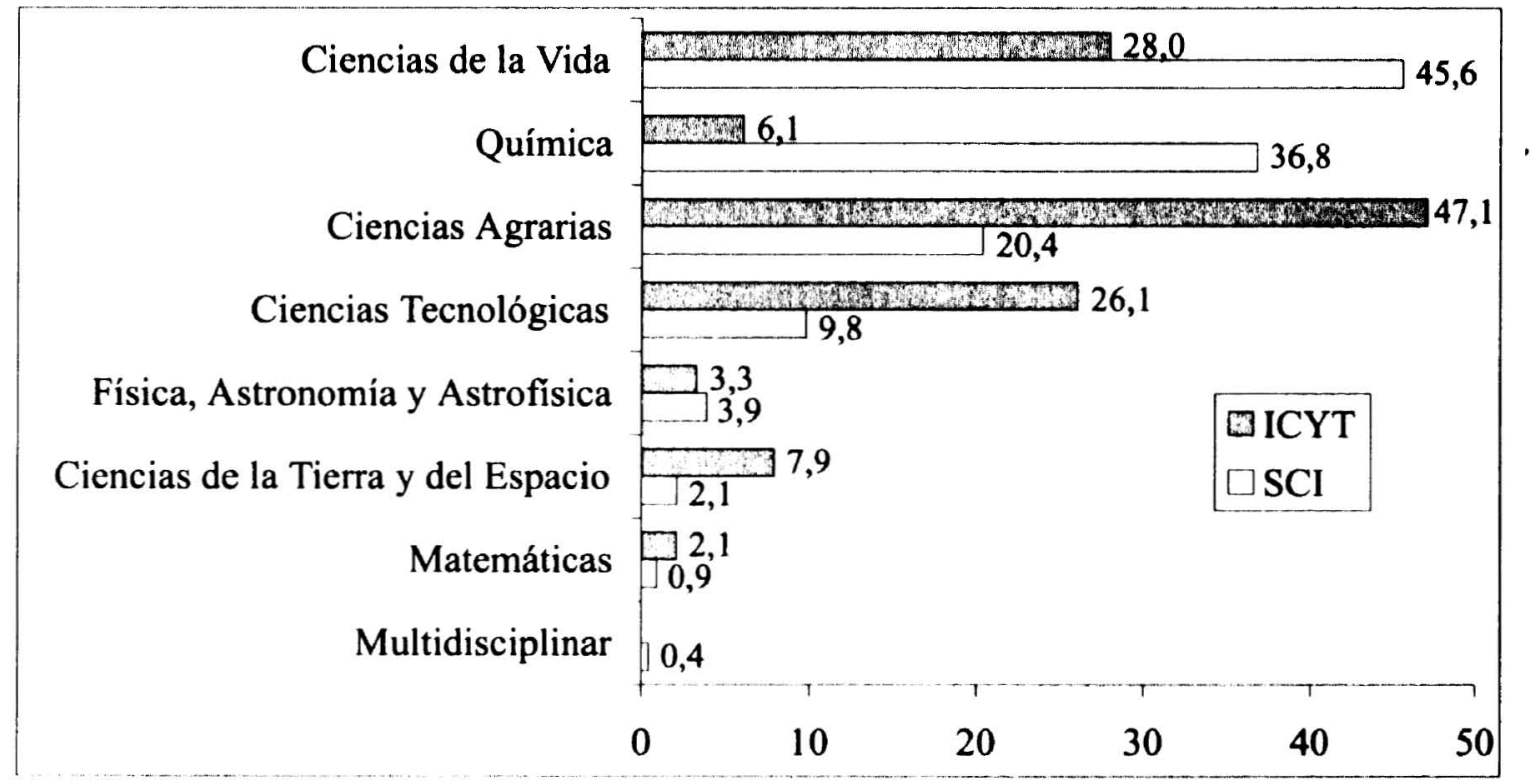


Figura 5

Distribución temática de documentos de la Universidad de Granada (en porcentaje)

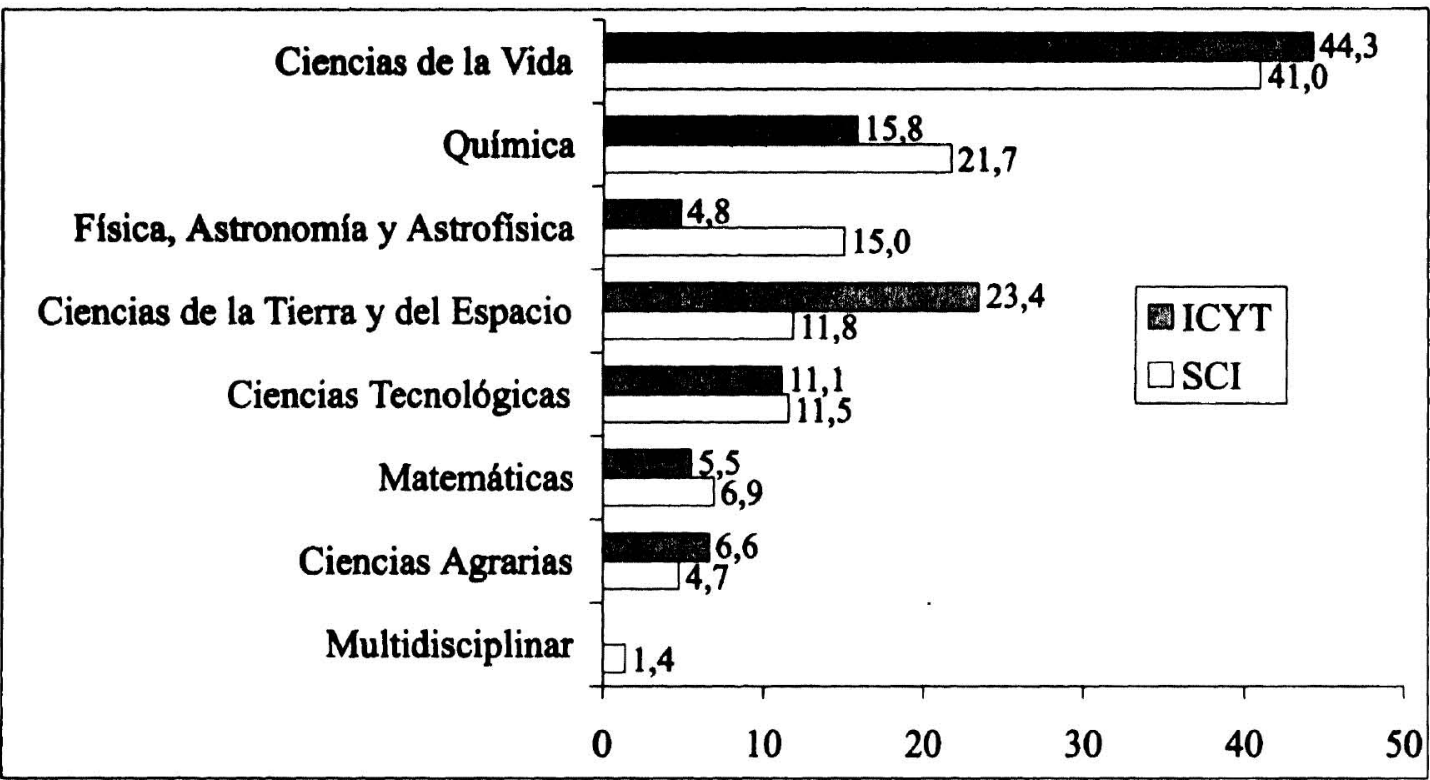

Figura 6

Distribución temática de documentos de la Universidad de Huelva (en porcentaje)

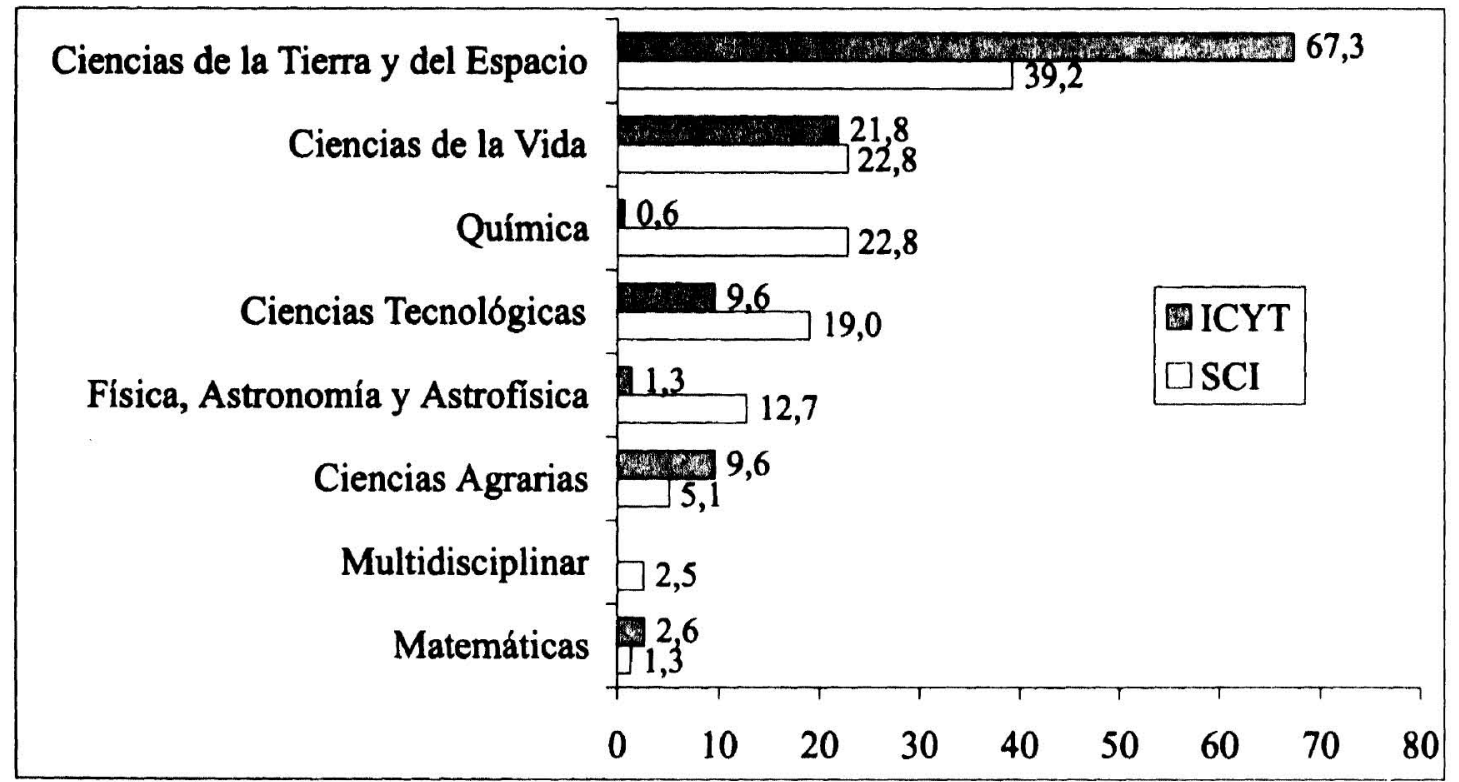




\section{Figura 7}

Distribución temática de documentos de la Universidad de Jaén (en porcentaje)

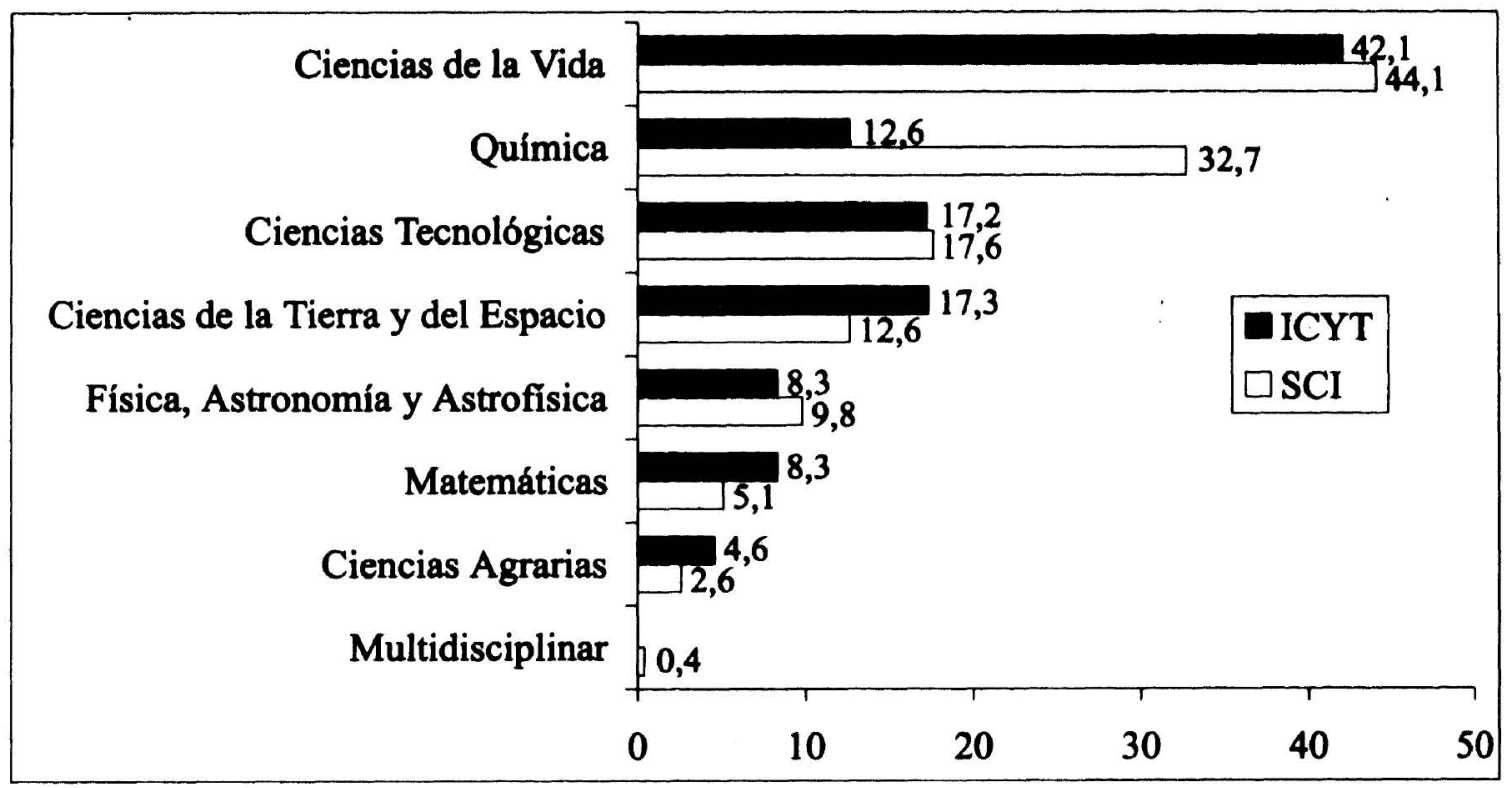

Figura 8

Distribución temática de documentos de la Universidad de Málaga (en porcentaje)

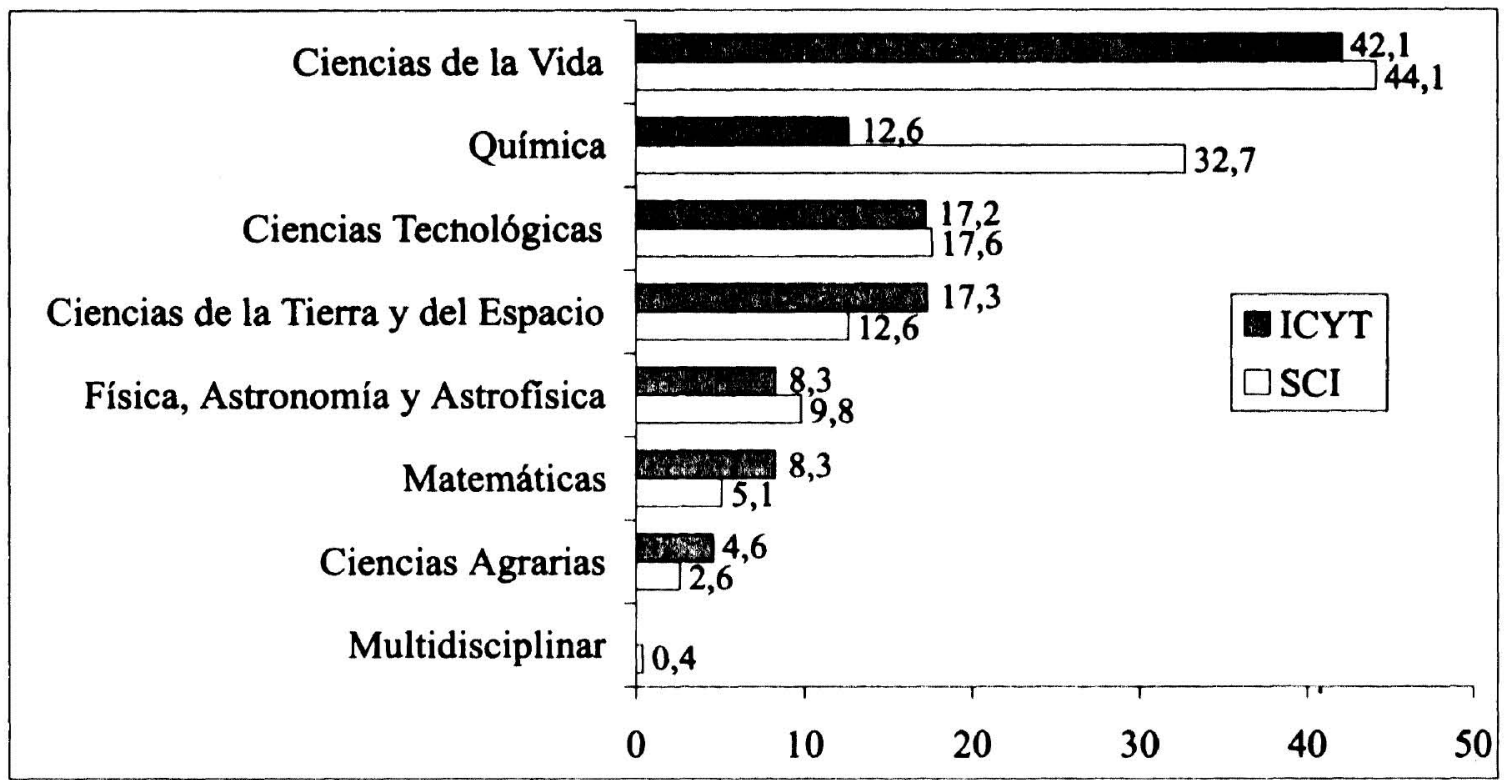


Figura 9

Distribución temática de documentos de la Universidad de Sevilla (en porcentaje)

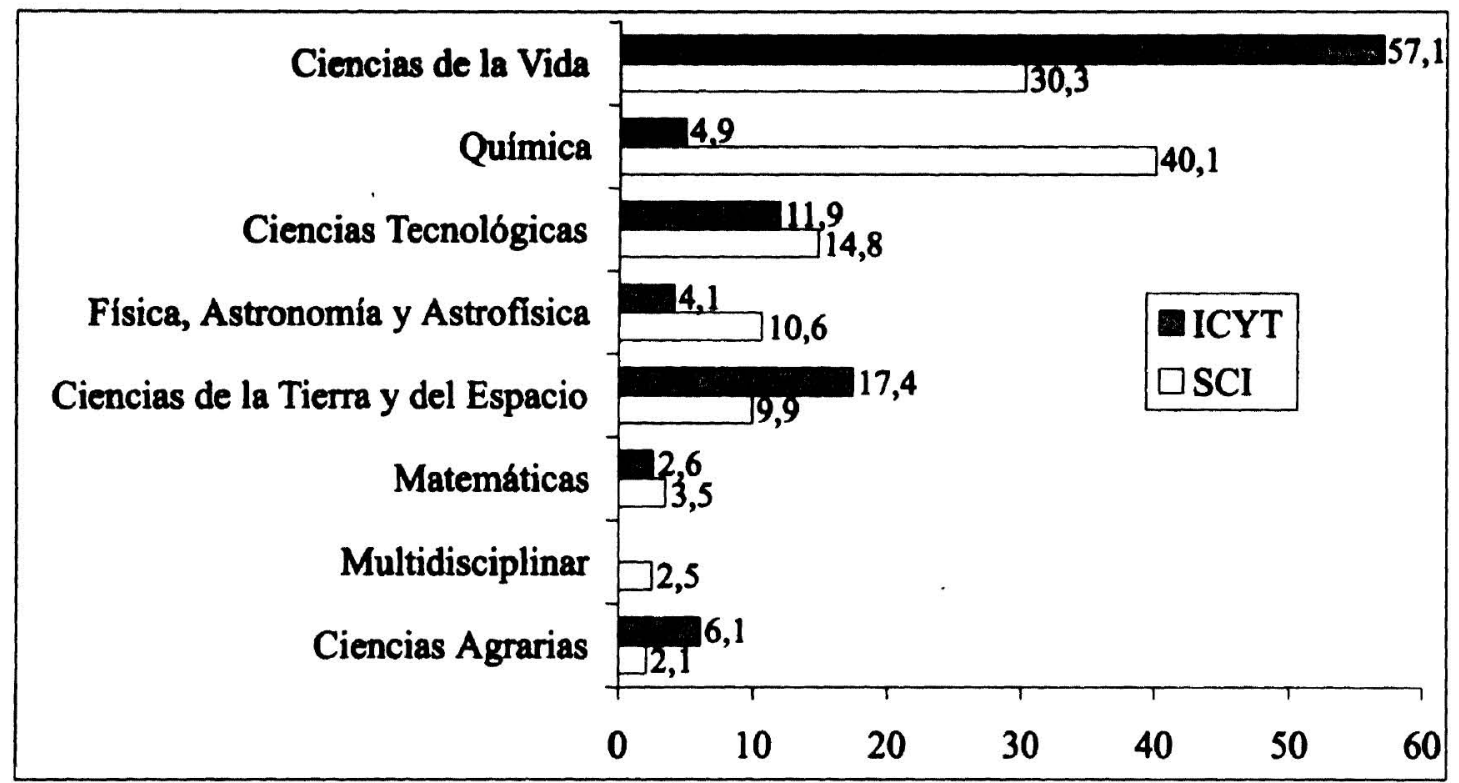

\section{Revistas}

En la base de datos SCI, los 12.530 documentos producidos por autores de organismos de Andalucía han sido publicados en 1.616 títulos de revistas. De éstos, 26 recogen más de 50 documentos (tabla VII). La revista más productiva es Analytica Chimica Acta, con 191 documentos, seguida de Astronomy and Astrophysics (179 documentos) y de Journal of Agricultural and Food Chemistry (121 documentos); las tres recogen artículos de investigación muy básica, como era de esperar en esta base de datos. No aparecen revistas editadas en España debido a que el SCI recoge muy pocas, en su mayoría de Medicina Clínica (aparte de Anales de Química que ha sido excluida deliberadamente del estudio para no solapar con la base de datos ICYT).

En la base de datos ICYT, los 11.070 documentos producidos por autores de organismos de Andalucía han sido publicados en 420 títulos de revistas españolas. La tabla VIII nos muestra las 24 revistas que recogen más de 100 trabajos pertenecientes a centros andaluces, que han difundido el $50 \%$ de los documentos de Ciencia y Tecnología generados en Andalucía. Las revistas más productivas han sido Anales de Química y Ars Pharmaceutica editadas respectivamente por la Real Sociedad Española de Química de Madrid y por la Facultad de Farmacia de la Universidad de Granada. Del conjunto de revistas que han publicado más de 100 artículos, 8 son editadas en la Comunidad Andaluza y representan el $20 \%$ de los documentos generados por los organismos de dicha comunidad autónoma. 
Tabla VII

Producción científica de Andalucía, desglosada por revistas (SCI)

\begin{tabular}{|c|c|}
\hline Título y editor & $N .^{o}$ documentos \\
\hline $\begin{array}{l}\text { Analytica Chimica Acta } \\
\text { (Elsevier Science Publishers, BV, Holanda) } \\
\text { Astronomy and Astrophysics } \\
\text { (Springer Verlag, Alemania) } \\
\text { Journal of Agricultural and Food Chemistry } \\
\text { (American Chemical Society, EE.UU.) } \\
\text { Analyst } \\
\text { (Royal Society of Chemistry, R. Unido) } \\
\text { Astrophysical Journal } \\
\text { (University of Chicago Press, EE.UU.) } \\
\text { Talanta } \\
\text { (Pergamon-Elsevier Science Ltd. R. Unido) } \\
\text { Carbohydrate Research } \\
\text { (Elsevier Science Publishers, BV, Holanda) } \\
\text { Phytochemistry } \\
\text { (Pergamon-Elsevier Science Ltd. R. Unido) } \\
\text { International Journal of Pharmaceutics } \\
\text { (Elsevier Science Publishers, BV, Holanda) } \\
\text { Thermochimica Acta } \\
\text { (Elsevier Science Publishers, BV, Holanda) } \\
\text { Fresenius Journal of Analytical Chemistry } \\
\text { (Springer Verlag, Alemania) } \\
\text { Tetrahedron } \\
\text { (Pergamon-Elsevier Science Ltd. R. Unido) } \\
\text { Physiologia Plantarum } \\
\text { (Munksgaard Intemational Publishers Ltd. Dinamarca) } \\
\text { Journal of Electroanalytical Chemistry } \\
\text { (Elsevier Science S.A. Lausana, Suiza) } \\
\text { Journal of Colloid and Interface Science } \\
\text { (Academic Press, Inc., Journal Division, EE.UU.) } \\
\text { Plant Physiology } \\
\text { (American Society of Plant Physiologists, EE.UU.) } \\
\text { Journal of Physical Chemistry } \\
\text { (American Chemical Society, EE.UU.) } \\
\text { Analytical Letters } \\
\text { (Marcel Dekker Journals, EE.UU.) } \\
\text { Journal of Physiology London } \\
\text { (Cambridge University Press, R. Unido) } \\
\text { Applied and Environmental Microbiology } \\
\text { (American Society for Microbiology, EE.UU.) } \\
\text { Journal of Chromatography A } \\
\text { (Elsevier Science Publishers, BV, Holanda) } \\
\text { Tetrahedron Letters } \\
\text { (Pergamon-Elsevier Science Ltd. R. Unido) } \\
\text { Electronics Letters } \\
\text { (I. E. E., R. Unido) } \\
\text { Nuclear Physics A } \\
\text { (Elsevier Science Publishers, BV, Holanda) } \\
\text { Physics Letters B } \\
\text { (Elsevier Science Publishers, BV, Holanda) } \\
\text { Journal of Bacteriology } \\
\text { (American Society for Microbiology, EE.UU.) }\end{array}$ & 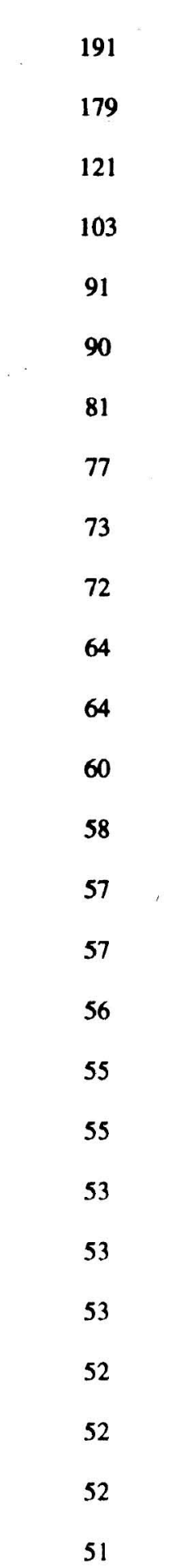 \\
\hline
\end{tabular}


Tabla VIII

Producción cientifica de Andalucía, desglosada por revistas (ICYT)

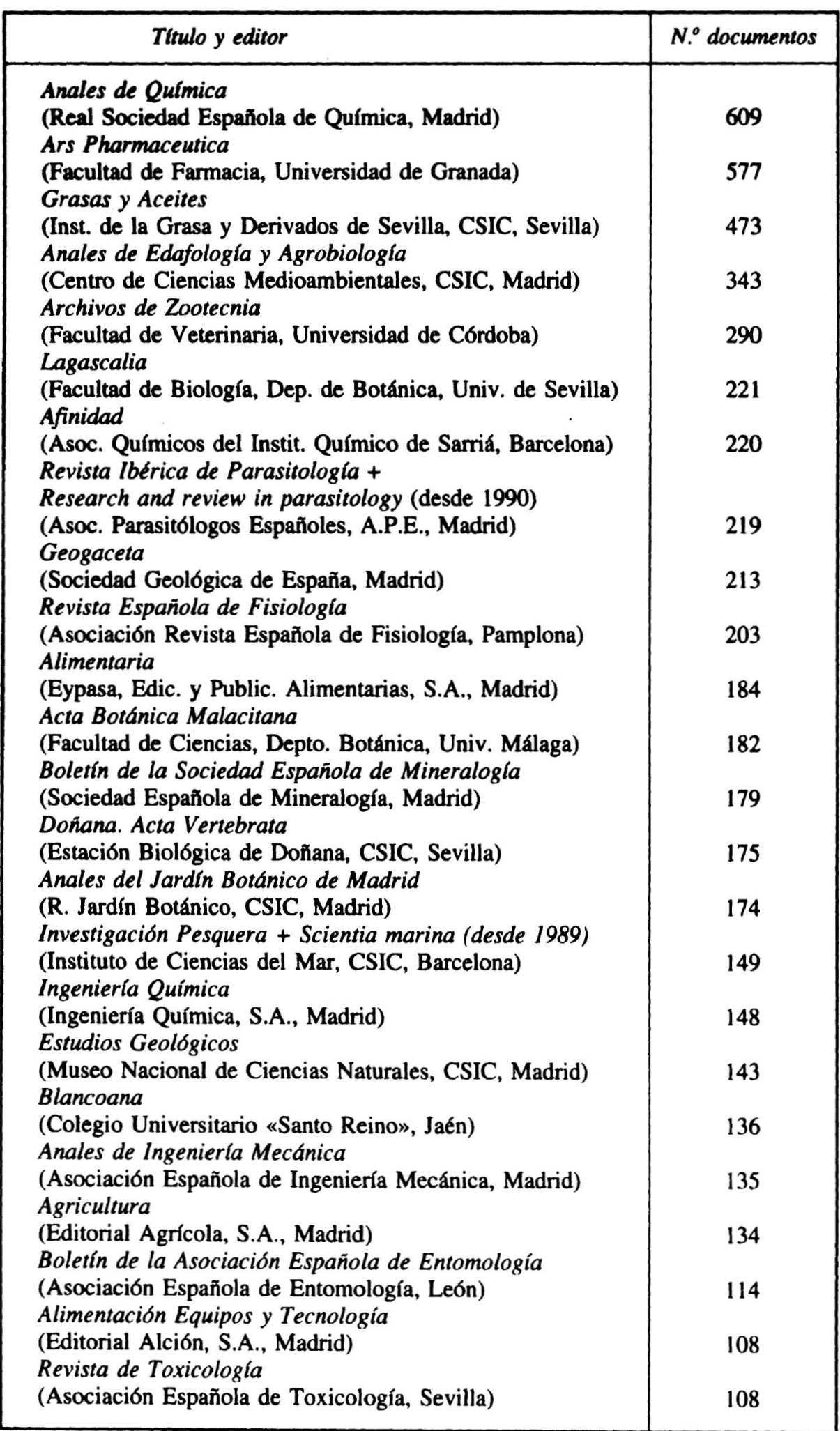




\section{Conclusiones}

A través de las bases de datos SCI e ICYT se observa que Andalucía aporta un 13-14\% de la producción científica total de España. Estos datos se refieren sólo a la producción en Ciencia y Tecnología publicada en revistas aparecidas en ambas bases de datos. Los datos de producción científica del área de la Medicina no se han recogido en el presente trabajo y para realizar el correspondiente análisis habría que recoger el campo de la Medicina clínica cubierto por el SCI para lo publicado internacionalmente y la base de datos IME (Índice Médico Español) (10) para lo publicado en revistas españolas.

Las provincias en las que se ha generado mayor número de trabajos han sido Granada y Sevilla, que suman entre las dos más del $60 \%$ de la producción de Andalucía. Destaca el peso que tiene en Andalucía la producción científica procedente de la Universidad (más del 75\%). El CSIC ocupa una segunda posición, con un $20 \%$ aproximadamente del total de documentos andaluces. Los organismos de investigación dependientes de la Junta de Andalucía han publicado alrededor de un $8 \%$ del total.

El campo científico sobre el que más trabajos se han publicado en Andalucía ha sido el de Ciencias de la Vida en las dos bases de datos, seguido del campo de la Química en la base de datos internacional y del campo de las Ciencias Tecnológicas en la base de datos nacional. Las ciencias básicas aparecen más representadas en la base de datos internacional ya que ésta recoge la denominada «corriente principal de la ciencia». La ciencia aplicada es de carácter local y aparece mejor representada en la base de datos ICYT.

Las revistas españolas que han publicado el mayor número de trabajos de autores de centros andaluces han sido Anales de Química y Ars Pharmaceutica. Entre las revistas internacionales destacan Analytica Chimica Acta y Astronomy and Astrophysics.

Por último, para conocer la producción científica andaluza en su conjunto, habría que completar el análisis que aquí se presenta con los datos procedentes de otras bases de datos internacionales, además de los de la base de datos de medicina española IME y los.de las áreas de Ciencias Sociales y Humanidades (ISOC).

\section{Bibliografía}

1. BASUlTO, J.; FRANCO, L.; SOLÍS, F. M.; VELASCO, F. (1995). Producción científica en Andalucía en las bases de datos del 1.S.I. 1990-1993. Junta de Andalucía. Plan Andaluz de Investigación. Universidad de Cádiz.

2. ORTEGA, C. (1984). Base de datos bibliográfica del Índice Español de Ciencia y Tecología. Actas de las Primeras Jornadas Españolas de Documentación Automatizada. Madrid, pp. 163-170.

3. Base de datos Science Citation Index en formato CD-ROM, años 1990-1997.

4. URDÍN, C.; VÁZQUEZ, M. (1997). Aportación de Andalucía a la producción científica española en Ciencia y Tecnología. I Jornadas Andaluzas de Documentación. Sevilla, 1314 de noviembre.

5. MORILlO, F.; FERNÁNDEZ, M. T.; GÓMEZ, I. (1997). Producción científica de Andalucía en la base de datos internacional Science Citation Index. I Jornadas Andaluzas de Documentación. Sevilla, 13-14 de noviembre.

6. VÁZQUEZ, M.; URDÍN, C. (1997). Los estudios sobre Galicia en las bases de datos del CSIC. Actas de las II Xornadas ANABAD Galicia, A Coruña, pp. 303-323. 
7. VÁZQUEZ, M.; URDÍN, C. (1996). Los estudios sobre Huelva publicados en revistas científicas españolas. Aestuaria (4), 141-165.

8. (1998) Investigación y desarrollo de la Comunidad de Madrid. Tres estudios sobre los recursos, producción y distribución de la actividad científica madrileña. Alfonso González Hermoso y José de la Sota (coord.). Dirección General de Investigación. Consejería de Educación y Cultura. Comunidad de Madrid.

9. Guía de la Universidad (1996). Ed. Consejo de Universidades. Secretaría General. Ministerio de Educación y Ciencia. Madrid.

10. OSCA LLUCH, J. (1998). La creación de bases de datos médicas en España: el Índice Médico Español y los índices de citas. En: Jornadas sobre Documentación y Ciencias Médicas. Zaragoza, Universidad de Zaragoza. 